\title{
ON ENTIRE FUNCTIONS DEFINED BY A DIRICHLET SERIES ${ }^{1}$
}

\author{
Q. I. RAHMAN
}

1. Introduction. Consider the Dirichlet series

$$
f(s)=\sum_{n=1}^{\infty} a_{n} e^{-8 \lambda_{n}}, \lambda_{n+1}>\lambda_{n}, \lambda_{1} \geqq 0, \lim _{n \rightarrow \infty} \lambda_{n}=\infty, s=\sigma+i t,
$$

where

$$
\lim _{n \rightarrow \infty} \inf \left(\lambda_{n+1}-\lambda_{n}\right)=h>0 .
$$

Let $\sigma_{c}$ and $\sigma_{a}$ denote, respectively, the abscissa of convergence and the abscissa of absolute convergence of $f(s)$. If $\sigma_{c}=-\infty, f(s)$ defines an entire function. I shall suppose throughout that $\sigma_{c}=\sigma_{a}=-\infty$.

Let

$$
\mu(\sigma)=\max _{n \geqq 1}\left|a_{n}\right| e^{-\sigma \lambda_{n}}, \quad M(\sigma)=\max _{-\infty<t<\infty}|f(\sigma+i t)| .
$$

It follows [1, p. 187] from hypothesis (1) that ${ }^{2} D<\infty$ and

$$
\limsup _{n \rightarrow \infty} \frac{\log n}{\lambda_{n}}=0
$$

For a positive $\epsilon$, one can choose therefore a positive integer $N(\epsilon)$ such that $\log n<(\epsilon / 2) \lambda_{n}$ for $n \geqq N(\epsilon)$. Consequently

$$
\begin{aligned}
M(\sigma) & \leqq \sum_{n=1}^{\infty}\left|a_{n}\right| e^{-\sigma \lambda_{n}} \\
& =\sum_{n=1}^{N(\epsilon)-1}\left|a_{n}\right| e^{-\sigma \lambda_{n}}+\sum_{N(\epsilon)}^{\infty}\left|a_{n}\right| e^{-(\sigma-\epsilon) \lambda_{n}} \cdot e^{-\epsilon \lambda_{n}}, \\
& <N(\epsilon) \mu(\sigma)+\mu(\sigma-\epsilon) \sum_{N(\epsilon)}^{\infty} \frac{1}{n^{2}} \\
& <K_{1} \mu(\sigma-\epsilon), \quad \sigma>\sigma_{a} .
\end{aligned}
$$

For a fixed $t_{0}$, let $S(R)$ denote the horizontal strip $\left|t-t_{0}\right|<R$. Put

$$
M_{S}(\sigma)=\underset{\left|t-t_{0}\right| \leqq R}{\operatorname{Max}}|f(\sigma+i t)|
$$

Received by the editors July 17, 1958.

1 Research supported by the National Science Foundation.

2 For notations see [1]. 
and let

$$
\lim _{\sigma \rightarrow-\infty} \sup _{\inf } \frac{\log \log M(\sigma)}{-\sigma}={ }_{\lambda}^{\rho} ; \quad \lim _{\sigma \rightarrow-\infty} \sup \frac{\operatorname{sinf} \log M_{S}(\sigma)}{-\sigma}={ }_{\lambda_{S}}^{\rho_{S}} .
$$

For $0<\rho<\infty$ and $0<\rho_{S}<\infty$, respectively, let

$$
\lim _{\sigma \rightarrow-\infty} \sup \frac{\log M(\sigma)}{e^{-\rho \sigma}}={ }_{\tau}^{T} ; \quad \lim _{\sigma \rightarrow-\infty} \sup \frac{\log M_{S}(\sigma)}{e^{-\rho_{S} \sigma}}=\begin{gathered}
T_{S} \\
\tau_{S}
\end{gathered} .
$$

Mandelbrojt and Gergen [1, pp. 219-220] have proved that the order $\rho_{S}$ of $f(s)$ in each horizontal strip $S(\pi a)$, with $a>D$, is equal to the order $\rho$ of $f(s)$. I prove here the following:

THEOREM 1. The lower order $\lambda_{S}$ of $f(s)$ in each horizontal strip $S(\pi a)$, with $a>D$, is equal to the lower order $\lambda$ of $f(s)$.

Theorem 2. If $h=\infty$, then the type $T_{S}$ and the lower type $\tau_{S}$ of $f(s)$ in each horizontal strip $S(\pi a)$, with $a>0$, satisfy respectively

$$
T_{S} \geqq e^{-\pi \rho a} T ; \quad \tau_{S} \geqq e^{-\pi \rho a} \tau .
$$

2. Proof of Theorem 1. If $S(\pi a)$ is the strip $\left|t-t_{0}\right|<\pi a$, and if

$$
M_{1}\left(\sigma_{j}+i t_{0}\right)=\operatorname{Max}|f(s)|
$$

when $s \in C\left(\sigma_{j}+i t_{0}, \pi a\right)$, we see [1, Theorem XVI] that, for an arbi$\operatorname{trary} \sigma_{j}$ :

(4) $\quad \log M_{1}\left(\sigma_{j}+i t_{0}\right) \geqq \log \left|a_{n}\right|-\log \Lambda_{n}-\lambda_{n} \sigma_{j}-K, \quad(n \geqq 1)$, where $K$ is a constant. Since $D<\infty$, we have [1, Theorem XVII]

$$
\limsup _{n \rightarrow \infty} \frac{\log \Lambda_{n}}{\lambda_{n}}<\infty \text {, }
$$

that is to say:

$$
\log \Lambda_{n}<P \lambda_{n}, \quad(n \geqq 1),
$$

where $P$ is a constant.

Let us denote by $s_{j}^{*}=\sigma_{j}^{*}+i t_{0}^{*}$ a quantity such that $M_{1}\left(\sigma_{j}+i t_{0}\right)$ $=\left|f\left(s_{j}^{*}\right)\right|$. It is obvious that $M_{S}\left(\sigma_{j}^{*}\right) \geqq M_{1}\left(\sigma_{j}+i t_{0}\right)$ and that $\sigma_{j}^{*}$ $=\sigma_{j}+k_{j}$, where $\left|k_{j}\right| \leqq \pi a$. The inequality (4) thus allows us to write

$$
\begin{aligned}
\log M_{S}\left(\sigma_{j}^{*}\right) & =\log M_{S}\left(\sigma_{j}+k_{j}\right) \\
& \geqq \log M_{1}\left(\sigma_{j}+i t_{0}\right) \\
& \geqq \log \left|a_{n}\right|-P \lambda_{n}-\lambda_{n} \sigma_{j}-K, \quad(n \geqq 1), \\
& =\log \left(\left|a_{n}\right| e^{-\left(\sigma_{j}+P\right) \lambda_{n}}\right)-K, \quad(n \geqq 1) .
\end{aligned}
$$


Consequently

$\log M S\left(\sigma_{j}^{*}\right) \geqq \log \mu\left(\sigma_{j}+P\right)-K>\log M\left(\sigma_{j}+P+\epsilon\right)-\log K_{1}-K$ by (3), and we get $\lambda_{S} \geqq \lambda$. But obviously $\lambda_{S} \leqq \lambda$, and therefore $\lambda_{S}=\lambda$.

Proof of Theorem 2. By the theorem of Mandelbrojt and Gergen [loc. cit.], since the conditions of that theorem are satisfied:

$$
\rho_{S}=\rho \text {. }
$$

If $h=\infty$, then [1, Theorem XVII]

$$
\limsup _{n \rightarrow \infty} \frac{\log \Lambda_{n}}{\lambda_{n}}=0,
$$

and for a positive $\epsilon^{\prime}$

$$
\log \Lambda_{n}<\epsilon^{\prime} \lambda_{n}, \quad(n \geqq 1) .
$$

Hence from (4), we have

$$
\begin{array}{rlrl}
\log M_{S}\left(\sigma_{j}^{*}\right) & =\log M_{S}\left(\sigma_{j}+K_{j}\right) \geqq \log M_{1}\left(\sigma_{j}+i t_{0}\right) & \\
& \geqq \log \left|a_{n}\right|-\epsilon^{\prime} \lambda_{n}-\lambda_{n} \sigma_{j}-K, \quad(n \geqq 1) \\
& =\log \left(\left|a_{n}\right| e^{-\left(\sigma_{j}+\epsilon^{\prime}\right) \lambda_{n}}\right)-K, \quad(n \geqq 1) .
\end{array}
$$

Consequently

$$
\begin{aligned}
\log M_{S}\left(\sigma_{j}^{*}\right) & =\log M_{S}\left(\sigma_{j}+k_{j}\right) \geqq \log \mu\left(\sigma_{j}+\epsilon^{\prime}\right)-K \\
& >\log M\left(\sigma_{j}+\epsilon^{\prime}+\epsilon\right)-\log K_{1}-K
\end{aligned}
$$

by (3), and we get

$$
\begin{aligned}
\frac{\log M_{S}\left(\sigma_{j}^{*}\right)}{e^{-\rho \sigma_{j}^{*}}} & =\frac{\log M_{S}\left(\sigma_{j}+k_{j}\right)}{e^{-\rho S\left(\sigma_{j}+k_{j}\right)}}>\frac{\log M\left(\sigma_{j}+\epsilon^{\prime}+\epsilon\right)}{e^{-\rho\left(\sigma_{j}+k_{j}\right)}}-\frac{\log K_{1}+K}{e^{-\rho\left(\sigma_{j}+k_{j}\right)}} \\
& =e^{\rho\left(\sigma_{j}-\epsilon^{\prime}-\epsilon\right)} \frac{\log M\left(\sigma_{j}+\epsilon^{\prime}+\epsilon\right)}{e^{-\rho\left(\sigma_{j}+\epsilon^{\prime}+\epsilon\right)}}-\frac{\log K_{1}+K}{e^{-\rho\left(\sigma_{j}+k_{j}\right)}} .
\end{aligned}
$$

Hence

$$
T_{S} \geqq e^{\rho\left(k_{j}-\epsilon^{\prime}-\epsilon\right)} T ; \quad \tau_{S} \geqq e^{\rho\left(k_{j}-\epsilon^{\prime}-\epsilon\right)} \tau,
$$

and, since $\left|k_{j}\right| \leqq \pi a$ and $\epsilon^{\prime}, \epsilon$ are arbitrary, we have

$$
T_{S} \geqq e^{-\pi \rho a} T ; \quad \tau_{S} \geqq e^{-\pi \rho a} \tau .
$$

\section{REFERENCE}

1. S. Mandelbrojt, Dirichlet series, Rice Inst. Pamphlet, vol. 31, no. 4 (1944).

NORTHWESTERN UNIVERSITY 